\title{
A Cohort of Gender-based Outcomes in Hepatitis B and C after Cardiac Surgery
}

\author{
Khushboo Nusrat ${ }^{1 *}$, Salar Anwar Molai ${ }^{1}$, Mahnoor Tahir ${ }^{1}$, Sayed Mustafa Shah', Mudassir Iqbal Dar ${ }^{1}$ \\ and Muhammad Farhan Khan ${ }^{1}$ \\ ${ }^{1}$ Dow University of Health Sciences, Karachi, Pakistan
}

\begin{abstract}
Objective: Our research article aimed to determine if six-month mortality amongst hepatitis B and C patients undergoing cardiac surgery varied according to gender, post-operatively. Secondarily, we highlighted the significant differences among the two genders in their pre-operative, operative, and post-operative characteristics and deduced significant predictors of mortality.

Methods: We obtained approval from the International Review Board of the Dow University of Health Sciences, and conducted a retrospective study targeting hepatitis B and C patients who had undergone cardiac surgery between January 2013 to October 2018 at the Ruth Pfau Civil Hospital, Karachi, Pakistan. The data was analysed using the Statistical Package for Social Sciences (Version 20.0). The population was divided into two groups, based on gender. Chi-squared test was used to compare categorical variables and odd ratios with $95 \%$ confidence interval were also computed. Differences in continuous variables were assessed using independent T-test or Mann-Whitney U test.
\end{abstract}

Results: There was no significant difference in six-month mortality between the genders, with a $22.5 \%$ mortality in males and $20.0 \%$ mortality in females. Post-operatively, males had higher creatinine $(p=0.003)$ levels but females tended to have a longer ward stay $(p=0.032)$. On multivariate logistic regression, duration of intubation (aOR=1.131, 95\% Cl: $1.002-1.275)$, cardiopulmonary bypass time $(\mathrm{aOR}=1.030,95 \% \mathrm{Cl}: 1.002-1.059)$ and duration of ward stay $(\mathrm{aOR}=1.100,95 \% \mathrm{Cl}: 1.031-1.175)$ were found to be significant predictors of mortality.

Conclusion: There is no association between six-month mortality and gender among hepatitis $\mathrm{B}$ and $\mathrm{C}$ patients undergoing cardiac surgery. Additionally, duration of intubation, cardiopulmonary bypass time and duration of ward stay are significant predictors of sixmonth mortality.

Keywords: Coronary artery bypass, coronary artery bypass, hepatitis $B$, hepatitis $C$, valvular heart disease.

\section{INTRODUCTION}

Chronic viral hepatitis is a debilitating condition, with approximately 400 million people being affected by the disease globally [1]. In Pakistan, $7.6 \%$ of the population is affected by hepatitis B and C, making it a disease which needs to be thoroughly researched [2]. Along with its direct effect on the liver, chronic viral hepatitis has been known to cause cardiovascular incidents owing to its' longstanding impact on blood vessels, ultimately resulting in atherosclerosis [3, 4]. Furthermore, studies have revealed how hepatitis $B$ and $C$ have an impact on mortality along with increased postoperative complications, in cardiac surgery patients $[5,6]$.

Keeping in view the influence of chronic viral hepatitis on cardiac patients, another study revealed greater mortality in females compared to males after an acute myocardial infarction in a population affected by this condition [7]. An increased mortality and morbidity in females undergoing cardiac surgery has also been documented [8]. At the same time, the lack of a significant difference in mortality due to gender has been noted [9]. These contradictions make it important to resolve whether the outcome of cardiac surgery amongst chronic viral hepatitis patients

\footnotetext{
*Corresponding Author: Khushboo Nusrat, Dow University of Health Sciences, Karachi, Pakistan; Email: khushboo.nusrat@gmail.com

Received: March 19, 2020; Revised: May 15, 2020; Accepted: June 10, 2020

DOI: https://doi.org/10.37184/lnjpc.2707-3521.1.25
}

varies according to gender, which will result in designing sex-specific post-operative interventions, ensuring minimal mortality in both males and females.

Considering the different outcomes of mentioned articles and lack of data, our primary objective was to investigate an association between post-operative six-month mortality, among a cohort of hepatitis patients undergoing cardiac surgery in a tertiary care hospital, and patient gender. Secondarily, we aimed to identify how preoperative, operative and post-operative characteristics differed between the two sexes. Furthermore, we aimed to assess the predictors of mortality in our population, thus adding to existing literature.

\section{MATERIALS AND METHODS}

A retrospective study was held in the city of Karachi, Pakistan at a tertiary care hospital over a period of six months from May to October 2018. The material was collected from the cardiac surgery ward at the Ruth Pfau Civil Hospital in Karachi, Pakistan. The data were obtained from discharge case files and extracted onto a standardised proforma which was obtained from senior doctors and customised according to our research. This study conforms to widely accepted ethical principles and was approved by the International Review Board of the Dow University of Health Sciences. Owing to the retrospective nature of our study, our ethical review board 
allowed us to access contact details of the patients, through the discharge case files. After explaining the purpose of the research, we obtained consent from the patients over the telephone.

\section{SELECTION AND DESCRIPTION OF PARTICIPANTS}

A retrospective study was conducted where we included all patients with hepatitis virus infection who underwent elective cardiac surgery at the Dr. Ruth Pfau Civil Hospital, Karachi from January 2013 to October 2018. A total of one hundred and sixteen patients who underwent valve replacement surgery or coronary artery bypass grafting were included, with normal liver function. We used anticipated frequency of $2.6 \%$ [8], population size of 1 million, confidence interval of $95 \%$ and $4 \%$ precision to calculate our sample size using openepi.com, which came to 61 . However, owing to the retrospective nature of our study, we had access to 116 patients in the given time frame. During data collection, we discarded all those files which had incomplete information, necessary for obtaining accurate results. Before the surgery was undertaken, the hepatitis profile, using PCR and other liver function tests were conducted. Our exclusion criteria consisted of those patients who had an abnormal liver profile, owing to the impact it would have on immediate outcomes, to reduce sampling bias. Additionally, we also discarded the case files which didn't have patient contact details, essential towards determining mortality at six months. The patient population encompassed all ages, ethnicity and religion.

\section{TECHNICAL INFORMATION}

The proforma was divided into four broad sections; patient information, pre-operative data, operative data and post-operative complications.

The patient information included their name, gender, smoking history and history of co-morbidities, specifically mentioning hypertension, rheumatic heart disease, diabetes, obesity, chronic obstructive pulmonary disease, liver dysfunction and atrial fibrillation along with details regarding hepatitis profile. The section on pre-operative data included all relevant cardiac information such as left ventricular function, left atrium size, systolic and diastolic dimensions. Relevant echo findings pointing toward any form of regurgitation or stenosis were also noted, along with the number of diseased vessels.

Operative data included procedural details namely cardiopulmonary bypass time, cross-clamp time, the size of the prosthetic valves used for valve replacements and the number of grafts used for the coronary artery bypass grafting. Post-operative complications such as wound infection, reopening, atrial fibrillation, any need for synchronised shock, low cardiac output was also assessed.

We aimed to assess the outcome by taking certain parameters into consideration. Operative data such as the use of an inotrope and an intra-aortic balloon pump were noted to determine their consequence on mortality. Amongst post-operative data, duration of intubation, intensive care unit (ICU) stay, ward stay, the need for blood transfusions, along with post-operative haemoglobin and creatinine were used to assess outcome. We contacted patients through phone calls, to determine six-month mortality.

\section{STATISTICAL ANALYSIS}

Data was analysed using the Statistical Package for Social Sciences (SPSS) (version 20.0). Categorical variables were expressed using frequencies and percentages. Continuous variables were presented as mean \pm standard deviations, or median (inter-quartile range) as appropriate. The study population was divided into two groups based on gender. Chi-squared test or Fisher-exact test was used to compare categorical variables between the two groups. Differences in continuous variables were assessed using independent T-test or Mann-Whitney $U$ test, as appropriate. A univariate logistic regression was conducted to identify variables independently associated with mortality at 6 months, and their crude odds ratios were calculated. Variables that had a near significant $(p<0.20)$ effect influence on mortality were included in a multivariable model to produce adjusted odds ratios. A $5 \%$ level of significance was used throughout the study.

\section{RESULTS}

One hundred and sixteen patients with documented viral hepatitis infection were included in the analysis. The study cohort comprised of $74(63.8 \%)$ males and $42(36.2 \%)$ females. Baseline and pre-operative characteristics for these patients, stratified by gender, are shown in Table 1. The mean age for all patients was 41.58 15.01 years, with a younger mean age among female subjects $(p=0.002)$. Males $(n=19,25.7 \%)$ were more likely than females $(n=1,2.4 \%)$ to report a previous history of smoking $(p=0.001)$. No genderspecific differences were observed with respect to previous history of diabetes $(p=0.804)$, previous history of myocardial infarction $(p=0.097)$ or previous history of hypertension $(p=0.704)$. Most patients were hepatitis C positive $(n=86,74.1)$, followed by hepatitis $B(n=26$, $22.4)$ and combined hepatitis $B$ and $C(n=4,3.4)$. Gender was not significantly associated with the type of hepatitis profile $(p=0.306)$.

Female patients were more likely to display lower preoperative haemoglobin levels versus males $(p<0.001)$. Males were more likely to present with a higher baseline creatinine, as compared to females $(p<0.001)$. Most patients had a good $(>60 \%)(n=46,39.7 \%)$ to moderate $(30-60 \%)(n=69,59.5 \%)$ left ventricular ejection fraction (LVEF). Pre-operative assessments of LVEF $(p=0.307)$, systolic dimension $(p=0.172)$, mean diastolic dimension $(p=0.584)$ and left atrial dimensions $(p=0.160)$ were not significantly different among males and females (Table 1). 
Table 1: Baseline and pre-operative study characteristics; stratified by gender.

\begin{tabular}{|c|c|c|c|c|}
\hline Demographic Characteristic & All Patients $(n=116)$ & Males $(n=74)$ & Females $(n=42)$ & p-value \\
\hline Age (years) \# & $41.58 \pm 15.01$ & $44.73 \pm 14.542$ & $36.02 \pm 14.338$ & ${ }^{* *} 0.002$ \\
\hline Body Mass Index (kg/m2) \# & $23.53 \pm 5.41$ & $24.01 \pm 5.37$ & $22.68 \pm 5.44$ & 0.207 \\
\hline Smoker (\%) & $20(17.2)$ & $19(25.7)$ & $1(2.4)$ & ${ }^{* *} 0.001$ \\
\hline History of Diabetes (\%) & $15(12.9)$ & $10(13.5)$ & $5(11.9)$ & 0.804 \\
\hline History of Myocardial Infarction (\%) & $7(6.0)$ & $2(2.7)$ & $5(11.9)$ & 0.097 \\
\hline History of Hypertension (\%) & $30(25.9)$ & $20(27.0)$ & $10(23.8)$ & 0.704 \\
\hline Hepatitis C (\%) & $86(74.1)$ & $52(70.3)$ & $34(81.0)$ & \multirow{3}{*}{0.306} \\
\hline Hepatitis B (\%) & $26(22.4)$ & $18(24.3)$ & $8(19.0)$ & \\
\hline Hepatitis B and C (\%) & $4(3.4)$ & $4(5.4)$ & $0(0.0)$ & \\
\hline Good Left Ventricular Ejection Fraction (>60\%) & $46(39.7)$ & $26(35.1)$ & $20(47.6)$ & \multirow{3}{*}{0.307} \\
\hline Moderate Left Ventricular Ejection Fraction (30-60\%) & $69(59.5)$ & $47(63.5)$ & $22(52.4)$ & \\
\hline Poor Left Ventricular Ejection Fraction $(<30 \%)$ & $1(0.9)$ & $1(1.4)$ & $0(0.0)$ & \\
\hline Body Mass Index (kg/m2) \# & $1.00(0.70-1.10)$ & $1.00(0.80-1.20)$ & $0.80(0.60-1.00)$ & ${ }^{* *}<0.001$ \\
\hline Pre-operative Haemoglobin (g/dl) † & $11.75(9.60-13.00)$ & $12.4(11.0-13.5)$ & $10.2(8.2-11.6)$ & ${ }^{* *}<0.001$ \\
\hline Systolic Dimension † & $35.00(31.00-40.00)$ & $36.00(32.00-40.00)$ & $35.00(30.00-39.25)$ & 0.172 \\
\hline Diastolic Dimension \# & $49.75 \pm 9.04$ & $50.11 \pm 8.65$ & $49.14 \pm 9.76$ & 0.584 \\
\hline Left Atrial Dimensions † & $45.00(37.00-50.00)$ & $41.00(36.50-49.50)$ & $45.00(39.75-55.00)$ & 0.160 \\
\hline
\end{tabular}

Among the one hundred and sixteen patients undergoing cardiac surgery; $45(38.8 \%)$ underwent coronary artery bypass grafting (CABG) and $62(53.4 \%)$ underwent valve replacement surgery (Table 2 ). Females were more likely to receive a shorter aortic cross-clamp time, as opposed to males $(p=0.018)$. noted with respect to gender, however, females tended to receive fewer grafts than men $(p=0.032)$. Patients undergoing valvular replacement included those suffering from mitral regurgitation (62.9\%), mitral stenosis $(66.1 \%)$, aortic regurgitation $(41.9 \%)$, aortic stenosis $(21.0 \%)$ and tricuspid regurgitation (64.5\%). Types of

Table 2: Operative Characteristics of patients undergoing CABG and Valve Replacement surgery; stratified by gender.

\begin{tabular}{|c|c|c|c|c|}
\hline Coronary Artery Bypass Grafting Details & All Patients $(n=45)$ & Males $(n=34)$ & Females $(n=11)$ & p-value \\
\hline No. of Vessels Diseased $\dagger$ & $3.00(3.00-3.00)$ & $3.00(3.00-3.00)$ & $3.00(2.00-3.00)$ & 0.421 \\
\hline No. of Grafts † & $3.00(3.00-4.00)$ & $3.00(3.00-4.00)$ & $3.00(2.00-3.00)$ & ${ }^{*} 0.032$ \\
\hline Valve Replacement Details & All patients $(n=62)$ & Males $(n=36)$ & Females $(n=26)$ & $p$-value \\
\hline Mitral Regurgitation & $39(62.9)$ & $23(63.9)$ & $16(61.5)$ & 0.850 \\
\hline Mitral Stenosis & $41(66.1)$ & $23(63.9)$ & $18(69.2)$ & 0.661 \\
\hline Aortic Regurgitation & $26(41.9)$ & $17(47.2)$ & $9(34.6)$ & 0.321 \\
\hline Aortic Stenosis & $13(21.0)$ & $7(19.4)$ & $6(23.6)$ & 0.729 \\
\hline Tricuspid Regurgitation & $40(64.5)$ & $21(58.3)$ & $19(73.1)$ & 0.231 \\
\hline Mitral Valve Size \# & $30.429 \pm 2.607$ & $31.303 \pm 2.506$ & $29.174 \pm 2.249$ & ${ }^{* *} 0.002$ \\
\hline Aortic Valve Size \# & $23.143 \pm 2.413$ & $24.556 \pm 1.667$ & $20.600 \pm 0.894$ & ${ }^{* *} 0.001$ \\
\hline Mitral Valve Area † & $1.200(0.900-3.000)$ & $1.175(0.825-3.750)$ & $1.250(0.975-1.950)$ & 0.536 \\
\hline
\end{tabular}

\#: variables expressed as mean \pm standard deviation, $\uparrow$ : variables expressed as median (inter-quartile range).

*: denotes significant at $p<0.05,{ }^{* *}$ : denotes significant at $p<0.01$.

Table 2 displays the operative characteristics of patients undergoing CABG $(n=45)$ and valve replacement surgeries $(n=62)$, respectively. The median number of diseased vessels and vessel grafts among patients undergoing CABG was 3 in each case. No significant difference in the number of diseased vessels was

Table 3: Post-operative study characteristics; stratified by gender. valvular diseases did not significantly differ by gender. The mean size of implanted mitral $(p=0.002)$ and aortic valves $(p<0.001)$ was smaller in women versus men.

Post-operative haemoglobin levels did not significantly differ between the two groups $(p=0.147)$ (Table 3). Post-

\begin{tabular}{l|c|c|c|c}
\hline Post-operative Characteristics & All Patients $(\mathbf{n = 1 1 1 )}$ & Males $(\mathbf{n = 7 4 )}$ & Females $(\mathbf{n = 4 2 )}$ & $\mathbf{p}$-value \\
\hline Post-operative Haemoglobin $(\mathrm{g} / \mathrm{dL}) \dagger$ & $9.20(8.30-10.40)$ & $9.450(8.500-10.575)$ & $9.050(8.075-9.925)$ & 0.147 \\
\hline Post-operative Creatinine $(\mathrm{mg} / \mathrm{dL}) \dagger$ & $0.90(0.70-1.20)$ & $1.000(0.800-1.250)$ & $0.750(0.600-1.025)$ & ${ }^{* *} 0.003$ \\
\hline Intensive Care Unit stay (days) $\dagger$ & $2.00(2.00-3.00)$ & $2.50(2.00-3.00)$ & $2.00(2.00-3.25)$ & 0.673 \\
\hline Ward Stay (days) $\dagger$ & $15.00(11.00-22.00)$ & $14.00(10.00-21.00)$ & $17.5(12.00-26.00)$ & ${ }^{* *} 0.032$ \\
\hline Six-month Mortality $(\%)$ & 21.6 & 22.5 & 20.0 & 0.756 \\
\hline
\end{tabular}

†: variables summarized as median (inter-quartile range). 
Table 4: Univariate and multivariable logistic regression-predictors of six-month mortality.

\begin{tabular}{|c|c|c|c|c|}
\hline \multirow{2}{*}{ Variable } & \multicolumn{2}{|c|}{ Univariate Logistic Regression } & \multicolumn{2}{|c|}{ Multivariable Logistic Regression } \\
\hline & Odds Ratio $(95 \% \mathrm{Cl})$ & p-value & Odds Ratio $(95 \% \mathrm{Cl})$ & p-value \\
\hline Age & $1.045(1.010-1.080)$ & ${ }^{*} 0.010$ & $1.050(0.999-1.103)$ & 0.053 \\
\hline Pre-operative Creatinine & $4.667(1.081-20.156)$ & ${ }^{*} 0.039$ & $4.703(0.638-34.693)$ & 0.129 \\
\hline Duration of Intubation & $1.146(1.058-1.242)$ & ${ }^{* *} 0.001$ & $1.131(1.002-1.275)$ & ${ }^{*} 0.046$ \\
\hline Inotrope Use & $1.032(1.001-1.064)$ & ${ }^{*} 0.041$ & $1.034(0.992-1.078)$ & 0.118 \\
\hline Aortic Cross-clamp Time & $1.024(1.008-1.041)$ & ${ }^{* *} 0.004$ & $0.969(0.928-1.012)$ & 0.161 \\
\hline Cardiopulmonary Bypass Time & $1.019(1.007-1.032)$ & ${ }^{* *} 0.002$ & $1.030(1.002-1.059)$ & ${ }^{*} 0.035$ \\
\hline Duration of Ward Stay & $1.068(1.021-1.118)$ & ${ }^{* *} 0.004$ & $1.100(1.031-1.175)$ & ${ }^{* *} 0.004$ \\
\hline
\end{tabular}

$\mathrm{Cl}$ : confidence interval, ${ }^{*}$ : denotes significant at $p<0.05,{ }^{* *}$ : denotes significant at $p<0.01$.

operative creatinine levels were significantly higher in males $(p=0.003)$. Female patients tended to have a longer duration of ward stay as compared to their male counterparts $(p=0.032)$. No such association was noted with respect to duration of the ICU stay $(p=0.673)$ (Table 3).

The overall 6-month mortality for the study cohort was $21.6 \%$. The unadjusted 6 -month mortality rate was $22.5 \%$ in males and $20.0 \%$ in females. This difference was not significant on univariate analysis $(p=0.756)$. The logistic model predicting 6-month mortality is shown in Table 4. Univariate logistic regression revealed age, pre-operative creatinine $(p=0.039)$, duration of intubation $(p=0.001)$, inotrope use (0.041), aortic cross-clamp time $(p=0.004)$, cardiopulmonary bypass time $(p=0.002)$ and duration of ward stay $(p=0.004)$ to be significantly associated with increased 6-month mortality, after the surgical procedure (Table 4). After performing multivariable logistic regression, only the following variables remained independently associated with increased six-month mortality: duration of intubation $(p=0.046)$, cardiopulmonary bypass time $(p=0.035)$ and duration of ward stay $(p=0.004)$ (Table 4). Female gender was not significantly associated with an increased risk of atrial fibrillation $(p=0.593)$.

\section{DISCUSSION}

Over the years, cardiac surgery has seen major advancements and immense research has been conducted in this field. Chronic viral hepatitis in itself is a major contributor to morbidity and mortality and has been known to have cardiovascular manifestations. Owing to this factor and a general lack in the existing literature, we felt a need to determine gender-based mortality and morbidity in hepatitis patients undergoing cardiac surgery [9].

In our population, we noticed females suffering from hepatitis at a younger age compared to males, findings which are consistent with a study conducted by MaleweKolou et al. [10]. As screening for viral hepatitis is a part of antepartum care, the condition is naturally detected sooner in females compared to males. As found in a study conducted in Kenya assessing gender differences in smoking, our study also noticed that men smoked more than women, possibly owing to society's perception of smoking amongst women as inappropriate and unacceptable behaviour [11].
The preoperative profiles of both genders in terms of having a history of hypertension and myocardial infarction didn't vary significantly which contrasts with a study where females had a greater incidence of hypertension and a lower incidence of myocardial infarction. In the same study, gender differences regarding diabetes were insignificant, a finding which was supported by our results as well [9]. Owing to our population having a mean age of forty-two years, the reason why we obtained insignificant findings in relation to the preoperative profiles could be that such conditions haven't been yet diagnosed in our patients. According to our results, female patients displayed lower haemoglobin levels compared to males. As women are known to have mean haemoglobin levels which are $12 \%$ lower than age and race-matched men due to underlying physiological phenomenon, this is unsurprising [12]. Male patients presented with higher creatinine levels, in line with previous findings where females tend to have lower serum creatinine levels than men, when kidneys have similar function [13]. Salton CJ. et al. found no considerable differences of left ventricular ejection fraction between men and women; which is consistent with our findings [14]. Additionally, other modalities of pre-operative assessment also remained insignificant between the two genders.

Intra-operative characteristics were taken into consideration to assess morbidity. However, out of aortic cross-clamp time, duration of intubation, usage of inotropes and cardiopulmonary bypass time, only aortic cross-clamp time differed significantly between males and females, with females having a shorter cross-clamp time. These findings are consistent in a study conducted in Australia [15] but inconsistent with another study conducted in Saudi Arabia [16].

The population we were taking into consideration consisted of those undergoing cardiac bypass surgery or valve replacement surgeries. Our results displayed that most of the patients undergoing CABG were those who had triple vessel disease. This finding doesn't come as a surprise as the preference of CABG over other treatment modalities such as percutaneous angiography, for triple vessel heart disease is well-documented [17]. Though we didn't observe a difference in the number of diseased vessels according to gender, our results displayed that females received fewer grafts than men, reinforced by similar findings [16]. Studies researching outcome between genders have repeatedly noticed the 
lesser use of the Left Internal Mammary Artery graft in women, which has ultimately led to worse outcome amongst women [18]. However, the varying number of grafts amongst the genders in our population seemingly had no effect on mortality, calling for further research regarding the relationship between graft number and outcome.

Though a higher incidence of mitral valve stenosis and mitral valve prolapse, especially in younger women for the latter is observed, we noticed no such trend in our study [19]. Alternatively, the equal incidence of aortic valve stenosis between men and women is welldocumented, like what we observed [19]. However, our female population tended to be implanted with a smaller prosthesis compared to males, possibly owing to the smaller Body Surface Area of females. A study investigating mortality in patients where a smaller prosthesis was used, also identified the female gender and a lower body surface area as conditions where a smaller prosthesis was employed for aortic valve replacement [20].

Our results displayed higher post-operative creatinine levels in males compared to females, a finding which is in disparity with what has been seen in other studies [21]. Similarly, the literature also states that a higher post-operative creatinine level should lead to a greater duration of the ward stay. Our results differed in this point too as they showed a longer ward stay in females, who, in our study had lower post-operative creatinine levels compared to males [21].

The six-month mortality in our population was $21.6 \%$, much higher compared to the $5 \%$ seen in a study conducted in the Netherlands [22] and the 3\% seen in a study carried out in Egypt [23]. As our population hails from a poor socioeconomic background, factors such as late presentation, more co-morbidities, and a weaker immune system may all contribute to our findings. Regardless of the cause for such disparity, methods to ensure that public have adequate awareness concerning heart disease and how it presents, coupled with better treatment strategies, all need to be employed to lessen the worrisome mortality rate seen in our population. Our study showed no significant difference in mortality between males and females, all of whom were infected with hepatitis $B, C$ or both. Our findings are in accordance with what was found in an Australian study aiming to assess the impact of gender on outcomes after cardiac surgery [9]. However, our study refutes what has been found in other studies which conclude that females tend to have greater morbidity [8]. Owing to these conflicting statements there is still a need to further investigate the impact of gender on mortality, amongst chronic viral hepatitis patients, especially in Pakistan where statistics show that nearly eight percent of the population is suffering from the condition [2].
Through our study, we also aimed to assess those factors which were significantly associated with greater sixmonth mortality. Through univariate logistic regression, we identified age as being a predictor of mortality, which reinforces the findings outlined by a cohort which explored the differences in mortality between two populations, one ranging between $60-75$ and one above 75. A major reason contributing to the results in the mentioned study was how patients part of the younger age group tended to undergo elective surgery instead of emergency treatment, compared to the older age group, a factor which could have seriously impacted our study as well [24]. We identified longer aortic cross-clamp time and cardiopulmonary bypass time as predictors of mortality, both of which were also identified in a study conducted in Brazil, with inotrope use also joining the two as a predictor [25]. Unsurprisingly, prolonged intubation was also a significant predictor of mortality, the same result being demonstrated in other studies, where hospital mortality was significantly higher compared to that seen at one year. Despite this difference, there was still a marked difference in mortality at one year for those patients who underwent prolonged intubation compared to those who didn't [26]. Interestingly we found ward stay to be a significant predictor of mortality, compared to prolonged ICU stay. Prolonged ward stay as a predictor is minimally researched, but those studies which have considered total length of stay (ICU and ward stay combined), have demonstrated its' greater impact on mortality compared to an average length of hospital stay [27]. On multivariate logistic regression, the significant predictors of mortality were lessened, consisting of prolonged ward stay, cardiopulmonary bypass time and prolonged intubation. Increased cardiopulmonary bypass time has been identified as a predictor of mortality in other studies as well [28] and prolonged intubation has also been associated with longer ICU stay which can lead to greater chances of mortality [29].

\section{CONCLUSION}

Our study showed no significant difference in mortality for hepatitis patients, based on gender. However, our study outlined certain predictors of mortality such as prolonged intubation time, prolonged ward stays and cardiopulmonary bypass time, adding to the current literature regarding cardiac surgery and factors affecting its' outcomes.

\section{LIMITATIONS}

This study was conducted in a single tertiary centre which limits the generalisability of our findings. Our study was limited due to the inclusion criteria employed. Furthermore, the sample was dominated by male subjects which may limit our ability to draw comparisons by gender. Despite these factors, our research provides vital insight in the outcomes observed after cardiac surgery. 


\section{CONFLICT OF INTEREST}

The author declares no conflict of interest.

\section{ACKNOWLEDGEMENTS}

Declared none.

\section{FUNDING}

There are no funding sources to declare.

\section{REFERENCES}

1. Data and statistics. Available from: http://www.euro.who.int/en/ health-topics/communicable-diseases/hepatitis/data-and-statistics (Accessed on: September 01, 2018).

2. Ashraf S, Ahmad A. Viral hepatitis in Pakistan: challenges and priorities. Asian Pac J Trop Biomed 2015; 5(3): 190-1.

3. Karsen H, Binici I, Sunnetcioglu M, Baran Al, Ceylan MR, Selek $\mathrm{S}$, et al. Association of paraoxonase activity and atherosclerosis in patients with chronic hepatitis B. Afr Health Sci 2012; 12(2): 114-8.

4. Adinolfi LE, Zampino R, Restivo L, Lonardo A, Guerrera B, Marrone $A$, et al. Chronic hepatitis $C$ virus infection and atherosclerosis: clinical impact and mechanisms. World J Gastroenterol 2014; 20(13): 3410

5. Sharma D, Sisodia A, Devgarha S, Mathur RM. Evaluation of early postoperative complications after open heart surgery in Hepatitis-B positive patients. Heart India 2016; 4(2): 56.

6. Hsieh WC, Chen PC, George G, Tinica G, Corciova FC. Prevalence of post-operative morbidity risk factors following cardiac surgery in patients with chronic viral hepatitis: a retrospective study. Hypertension 2015; 68: 66-7.

7. Kuo PL, Lin KC, Tang PL, Cheng CC, Huang WC, Chiang $\mathrm{CH}$ et al. Contribution of hepatitis $\mathrm{B}$ to long-term outcome among patients with acute myocardial infarction: a nationwide study. Medicine 2016; 95(5): e2678.

8. Filardo G, Hamman BL, Pollock BD, da Graca B, Sass DM, Phan $\mathrm{TK}$, et al. Excess short-term mortality in women after isolated coronary artery bypass graft surgery. Open Heart 2016; 3(1): e000386.

9. Saxena A, Poh CL, Dinh DT, Reid CM, Smith JA, Shardey GC et al. Does patient gender affect outcomes after concomitant coronary artery bypass graft and aortic valve replacement? An Australian Society of Cardiac and Thoracic Surgeons Database study. Cardiology 2011; 119(2): 116-23.

10. Kolou M, Katawa G, Salou M, Gozo-Akakpo KS, Dossim S, Kwarteng A, et al. High prevalence of hepatitis B virus infection in the age range of $20-39$ years old individuals in Lome. Open Virol $\mathrm{J}$ 2017; 11: 1-7

11. Kaplan M, Carriker L, Waldron I. Gender differences in tobacco use in Kenya. Soc Sci Med 1990; 30(3): 305-10.

12. Murphy WG. The sex difference in haemoglobin levels in adultsmechanisms, causes, and consequences. Blood Rev 2014; 28(2): 41-7.

13. O'Leary JG, Wong F, Reddy KR, Garcia-Tsao G, Kamath PS, Biggins SW, et al. Gender-specific differences in baseline, peak, and Delta serum creatinine: the NACSELD experience. Dig Dis Sci 2017; 62(3): 768-76

14. Salton CJ, Chuang ML, O'Donnell CJ, Kupka MJ, Larson MG, Kissinger KV, et al. Gender differences and normal left ventricular anatomy in an adult population free of hypertension: A cardiovascular magnetic resonance study of the Framingham Heart Study Offspring cohort. J Am Coll Cardiol 2002; 39(6): 105560.
15. Saxena A, Poh CL, Dinh DT, Reid CM, Smith JA, Shardey GC, et al. Early and late outcomes after isolated aortic valve replacement in octogenarians: an Australasian Society of Cardiac and Thoracic Surgeons Cardiac Surgery Database Study. Eur J Cardiothorac Surg 2012; 41(1): 63-8.

16. Ahmad M, Arifi AA, van Onselen R, Alkodami AA, Zaibag M, Khaldi $A A$, et al. Gender differences in the surgical management and early clinical outcome of coronary artery disease: Single centre experience. J Saudi Heart Assoc 2010; 22(2): 47-53.

17. Hawkes AL, Nowak M, Bidstrup B, Speare R. Outcomes of coronary artery bypass graft surgery. Vasc Health Risk Manag 2006; 2: 477-84.

18. Arif R, Farag M, Gertner V, Szabo G, Weymann A, Veres G, et al. Female gender and differences in outcome after isolated coronary artery bypass graft surgery: does age play a role? PLoS One 2016; 11(2): e0145371.

19. Women \& Valve Disease. Available from: https://my.clevelandclinic. org/health/diseases/17647-women--valve-disease (Accessed on: September 01, 2018).

20. LaPar DJ, Ailawadi G, Bhamidipati CM, Stukenborg G, Crosby IK, Kern JA, et al. Small prosthesis size in aortic valve replacement does not affect mortality. Ann Thorac Surg 2011; 92(3): 880-8.

21. Najafi M. Serum creatinine role in predicting outcome after cardiac surgery beyond acute kidney injury. World J Cardiol 2014; 6(9): 1006.

22. Siregar S, Groenwold RH, de Mol BA, Speekenbrink RG, Versteegh $\mathrm{MI}$, Brandon $\mathrm{B}$, et al. Evaluation of cardiac surgery mortality rates: 30-day mortality or longer follow-up?. Eur J Cardiothorac Surg 2013; 44(5): 875-83.

23. Khalifa YS, Eisa KM, Bary MA, Ismail HE, Taha AM. Short term outcome of coronary artery bypass graft surgery: evaluation of recently established cardiac center. J Egypt Soci Cardiothorac Surg 2018; 26(1): 24-9.

24. Naughton C, Feneck RO, Roxburgh J. Early and late predictors of mortality following on-pump coronary artery bypass graft surgery in the elderly as compared to a younger population. Eur $\mathrm{J}$ Cardiothorac Surg 2009; 36(4): 621-7.

25. Fortes JV, Baldez TE, Costa MD, Silva LN, Pinheiro RS, Fecks ZS et al. Mortality risk after cardiac surgery: application of Inscor in a university hospital in Brazil's northeast. Braz J Cardiovasc Surg 2016; 31(5): 396-9.

26. Pappalardo F, Franco A, Landoni G, Cardano P, Zangrillo A, Alfieri $O$. Long-term outcome and quality of life of patients requiring prolonged mechanical ventilation after cardiac surgery. Eur $\mathrm{J}$ Cardiothorac Surg 2004; 25(4): 548-52.

27. Mounsey JP, Griffith MJ, Heaviside DW, Brown AH, Reid DS Determinants of the length of stay in intensive care and in hospital after coronary artery surgery. Heart 1995; 73(1): 92-8.

28. Salis S, Mazzanti VV, Merli G, Salvi L, Tedesco CC, Veglia F, et al. Cardiopulmonary bypass duration is an independent predictor of morbidity and mortality after cardiac surgery. J Cardiothorac Vasc Anesth 2008; 22(6): 814-22.

29. Azarfarin R, Ashouri N, Totonchi Z, Bakhshandeh H, Yaghoubi A Factors influencing prolonged ICU stay after open heart surgery. Res Cardiovasc Med 2014; 3(4): e20159. 